\title{
Track Damage Recognition Algorithm Based on Improved Convolution
}

\author{
Qiang JIA, Feng $\mathrm{HAN}^{1}$, Yuan LI, Miaomiao QI, and Zhibo LIU \\ College of Civil Engineering, Lanzhou Jiaotong University, Lanzhou 730070, China
}

\begin{abstract}
Railway inspection and maintenance in uninhabited high-cold areas are facing great challenges according to the rapid development of Sino-Russian railway. In this paper, an algorithm is proposed, which is image edge detection track structure recognition and damage detection based on improved convolution model. This paper use image acquisition method and improved two-dimensional convolution to image filtering, thus the original image matrix is processed by determinant transformation to enhance image boundary elements. On the basis of the linear characteristics obtained from edge detection, the fastener cartridge and steel rails are identified. The damage and position of steel rails are judged by the sharp change of alignment. The image recognition and verification of the existing railway demonstrates that the method has the following advantages: It improves the rate of recognition structure and has certain adaptability. At the same time, the corresponding position of the structure can be determined, which is beneficial to the identification of structural damage. Besides, it plays an important role in the daily operation and maintenance of track.
\end{abstract}

Keywords. Improve 2D convolution, edge detection, structure recognition, rail damage

\section{Introduction}

With the rapid, innovated and sustainable development of modern Sino-Russian railways, transportation demand is urgent. Thus railways have become an important link in operation, and tracks are the basis for locomotive operation therein. It is very important for the railway department to quickly and accurately identify the track structure and the specific location of the track damage and to repair according to the node and complete the maintenance in the environmental conditions of the uninhabited high-cold areas [1-4]. Combining industrial camera with the locomotive and collecting the track structure image for processing, it can effectively solve the track damage identification and repair \& maintenance under the difficult external conditions in the uninhabited high-cold areas.

The quantization of image edge grayscale in calculus is equivalent to the difference of discrete series or directional derivatives in a continuous function [5]. Canny [6-8] firstly proposed a multilevel edge detection algorithm. Lixia Liu [9] and others introduced guided filtering to smooth the image and find the threshold by the law. Min Yongzhi [10] and others proposed various scales of Retinex combined with surface inspection systems to enhance image preprocessing methods. However, the

${ }^{1}$ Corresponding Author, Feng Han, Shanxi, PhD, professor. E-mail: 153025377@qq.com. 
algorithm for identifying track structure damage based on the linear change of edge detection is still not involved. This paper preprocesses the acquired track images in high altitudes, uses improved convolution for image filtering, combines it with edge detection, compares and analyzes the processing results with the existing detection methods, and recognizes the partial track structure and judges the damage according to the alignment.

In this paper, the high-definition linear array camera (Figure 1) is used for image acquisition, with effective pixels up to 120 million pixels. The Photo acquisition is collected under the condition of auxiliary light source. A forthcoming operating line and an existing freight transport line are selected to collect photos of curved sections and track structures.

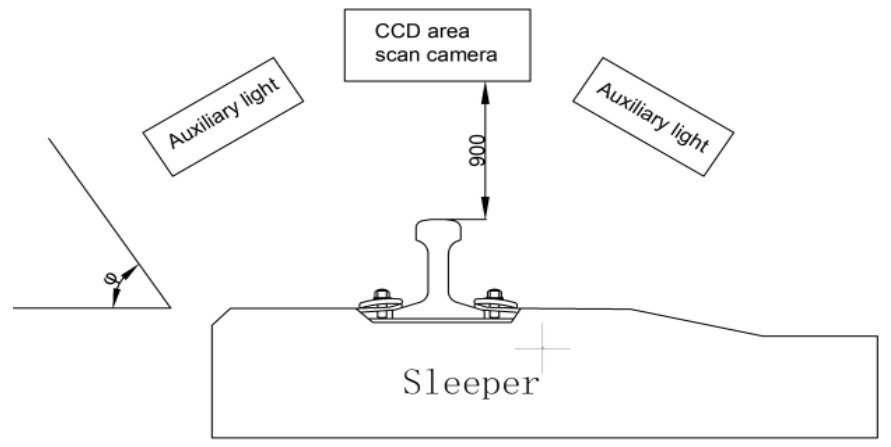

Figure 1. Schematic diagram of acquisition device

\section{Image Preprocessing}

\subsection{Image Gray Histogram and Equalization}

The changes of the pixel gray-level image are random, and the corresponding one-dimensional histogram graph is uneven. This process requires histogram equalization. This paper uses Opencv3 programming to process track images. OpenCV3 is the use of $\mathrm{C}++$ language programming and can be combined with MATLAB image processing, mainly used in machine vision and image processing. In OpenCV3, a certain algorithm is used to make the histogram roughly flat.

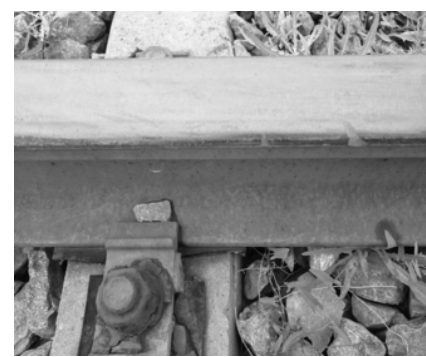

(a) Original image

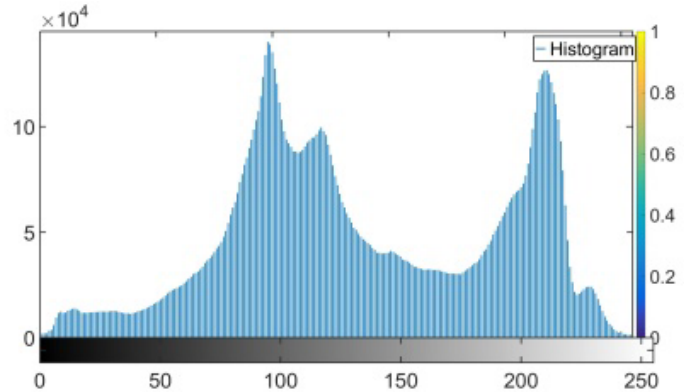

(b) One-dimensional histogram 

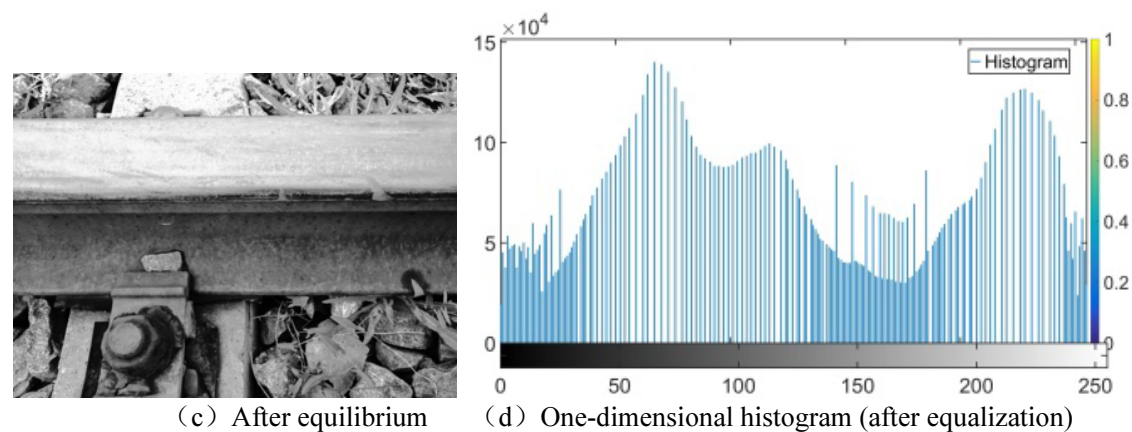

(d) One-dimensional histogram (after equalization)

Figure2. Image comparison after histogram equalization

The result is shown in Figure 2. It can be seen from the comparison that the process of equalization makes the gray scale harmonized, and the contrast is reduced to a certain extent; from the detailed histogram, it can be seen that the longitudinal height of the equalized histogram has been partially adjusted, and the gray value interval is effectively used, making the image expressive more expressive.

\subsection{Image Smoothing}

Smoothing is mainly used to reduce the distortion and a series of noise existing on the image [11-14]. Neighborhood filtering (convolution) is to combine pixels in a small neighborhood with different weights to perform weighted summation processing on the input pixels to obtain the pixel output value of the corresponding image. The specific process is shown in Figure 3, the left image and the middle image are convolved to produce the right image.

\begin{tabular}{|l|l|l|l|l|}
\hline 4 & 7 & 7 & 6 & 3 \\
\hline 5 & 4 & 9 & 8 & 1 \\
\hline 3 & 3 & 6 & 9 & 7 \\
\hline 2 & 4 & 5 & 7 & 4 \\
\hline 5 & 6 & 6 & 6 & 3 \\
\multicolumn{5}{c}{$f(x, y)$}
\end{tabular}

\begin{tabular}{|l|l|l|}
\hline 1 & 1 & 1 \\
\hline 1 & 2 & 1 \\
\hline 1 & 1 & 1 \\
\hline
\end{tabular}

$h(x, y)$

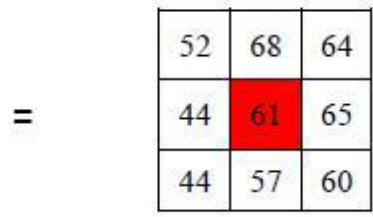

$g(x, y)$

Figure 3. Neighborhood filtering (convolution)

This paper proposes an improved convolutional image smoothing method. Through matrix determinant change, the unprocessed elements at the edge of the original image matrix are convolved to obtain the corresponding processing results. The specific improvement processes are:

- $\quad$ Fill the upper and left sides of the matrices $f$ and $h$ with 0 elements and increase their size to $\mathrm{M} \times \mathrm{N}$. The new matrices are named $f^{\prime}$ and $h^{\prime}$, which are simply expressed as formula (1):

$$
f^{\prime}(m, n)=\left\{\begin{array}{ll}
f(m, n), 0 \leq m \leq M_{1}, 0 \leq n \leq N_{1} \\
0, & \text { else }
\end{array} \quad h^{\prime}(m, n)= \begin{cases}h(m, n), 0 \leq m \leq M_{2}, 0 \leq n \leq N_{2} \\
0, & \text { else }\end{cases}\right.
$$


Stack $f^{\prime}$ in rows to reconstruct a $1 \times\left(\mathrm{M}^{*} \mathrm{~N}\right)$ row vector $f_{m}^{r}$. Specifically: after stacking the second row of $f^{\prime}$ on the first row, arrange the other rows in turn;

- Construct each row of $h^{\prime}$ to obtain an $\mathrm{N} \times \mathrm{N}$ circulant matrix, and use the circulant matrix as the "unit block matrix" to construct the circulant block matrix; the brief expression is as formula (2);

$$
D_{m}=\left(\begin{array}{cccc}
h^{\prime}(m, 0) & h^{\prime}(m, N-1) & \cdots & h^{\prime}(m, 1) \\
h^{\prime}(m, 1) & h^{\prime}(m, 0) & \cdots & h^{\prime}(m, 2) \\
\vdots & \vdots & \vdots & \vdots \\
h^{\prime}(m, N-1) & h^{\prime}(m, N-2) & \cdots & h^{\prime}(m, 0)
\end{array}\right) \quad D=\left(\begin{array}{cccc}
D_{0} & D_{1} & \cdots & D_{M-1} \\
D_{M-1} & D_{0} & \cdots & D_{M-2} \\
\vdots & \vdots & \vdots & \vdots \\
D_{1} & D_{2} & \cdots & D_{0}
\end{array}\right)
$$

The circulant block matrix $\mathrm{D}\left(\left(\mathrm{M}^{*} \mathrm{~N}\right) \times\left(\mathrm{M}^{*} \mathrm{~N}\right)\right)$ and the row vector $f_{\mathrm{m}}^{!}$are convolved in two, the matrix form is $G=f_{m}^{r} \cdot D$; The result is $1 \times\left(\mathrm{M}^{*} \mathrm{~N}\right)$ row vector, rearrange it into an $\mathrm{M} \times \mathrm{N}$ matrix $\mathrm{G}^{\prime}$;

- Determinant transformation processing is adopted for $G^{\prime}$, and the processing principle is "left row and right column" to obtain the final improved convolution image processing matrix $g$, and the expression is formula (3).

$$
g=\left(\begin{array}{cccc}
0 & 0 & \cdots & 1 \\
1 & 0 & \cdots & 0 \\
\vdots & \vdots & \vdots & \vdots \\
0 & 0 & \cdots & 0
\end{array}\right) \cdot\left(\cdot \cdot\left(\begin{array}{cccc}
0 & 1 & \cdots & 0 \\
0 & 0 & \cdots & 0 \\
\vdots & \vdots & \vdots & \vdots \\
1 & 0 & \cdots & 0
\end{array}\right)\right.
$$

The rail image is processed by improved convolution filtering and Gaussian filtering, and the program is written through OpenCV3.

Table 1.Comparison of image smoothing indicators

\begin{tabular}{cccc}
\hline & Figure 4 $(\mathrm{a})$ & Figure 4 $(\mathrm{b})$ & Figure 4 $(\mathrm{c})$ \\
\hline Time $(\mathrm{ms})$ & 783 & 781 & 688 \\
Clarity & 7.263 & 8.032 & 9.221 \\
\hline
\end{tabular}

The resulting linear filtering process can adjust the kernel value to obtain the denoising result. The experimental results are shown in Figure 4.

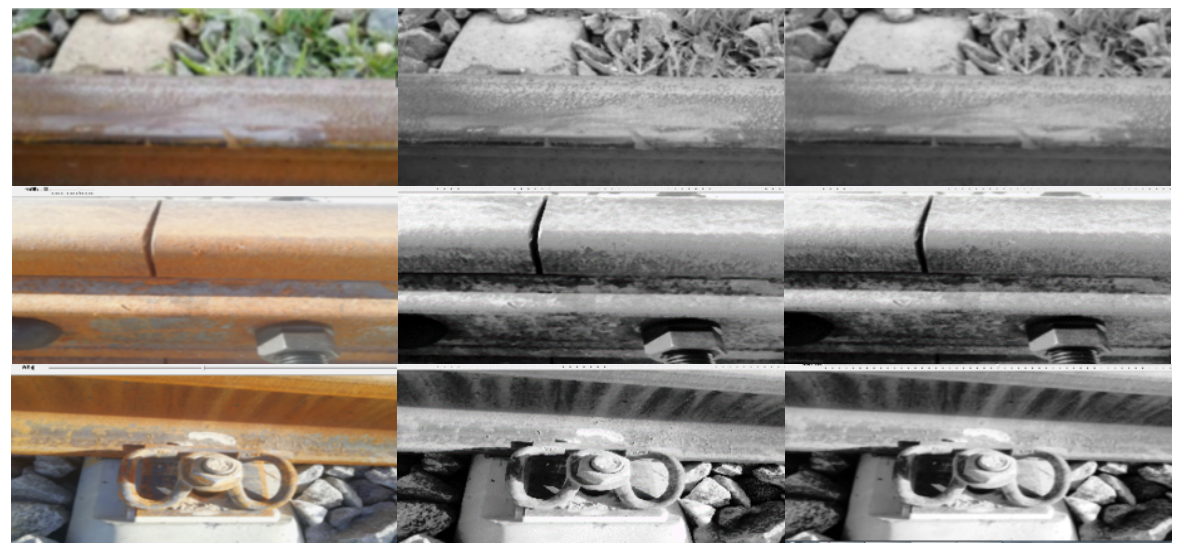

(a) Gaussian filtering

(b) Retinex method

(c) Method filtering in this article

Figure 4. Comparison of image filtering results

From the Figure 4, the comparison results are: for Gaussian filtering, when the 
kernel value is fixed (can be adjusted with the kernel value progress bar), while removing the noise of the outer parts of the track, the detail part of the image is also greatly affected; However, the smoothing method [11] in the literature has better detail processing results, but the loss of information on image edge position is more serious. The improved convolution filter proposed in this paper can better preserve the edge information of the image, and obtain better results in the image filter process at the same time. From the smoothing index in Table 1, this method reduces the matrix transposition process, improves the processing rate, retains the image edge information and has relatively high definition.

\section{Improve Edge Detection}

Edge detection needs to be filtered, enhanced and detected. In this paper, an improved edge detection algorithm combining linear change with edge detection is proposed. The algorithm flowchart is shown in Figure 5. The main process of the algorithm is as follows:

- Obtain the original image and load it into the file under the algorithm operation;

- Perform histogram equalization processing on the imported processed image, and output the processed renderings;

- Improved two-dimensional convolution filtering is used to smooth the image and the cutoff threshold is used to segment the threshold range. The threshold can be adjusted adaptively throughout the process;

- Obtain the edge line of the image, use the linear characteristics of the outer edge of the rail and the unique " $\omega$ " linear shape of the fastener cartridge for discriminant analysis, separate the fastener structure while identifying the rail, and perform linear discrimination on the surface of it. Partial damages on the rail surface can be determined if there is mutation on linear alignment, if not, it will output edge detection image.

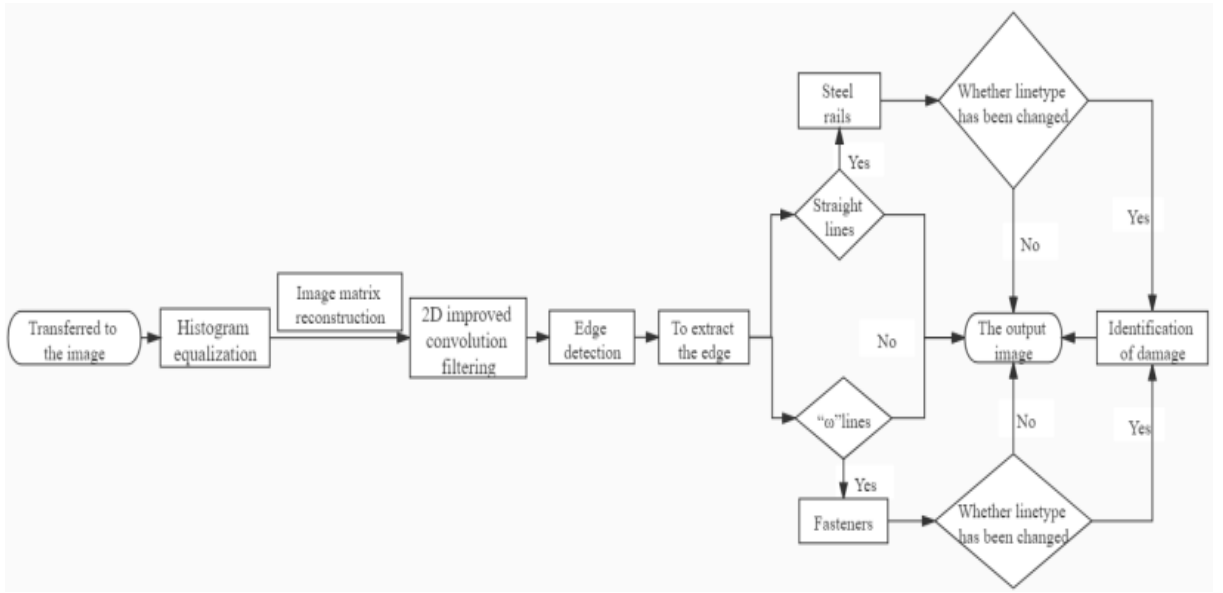

Figure 5. Improve edge detection algorithm flowchart 


\section{Track Verification}

In this paper, the authors perform image processing on the photos of rail joints and curve sections, image edge detection processing on rail joints and identify the elastic strips of the fasteners and separate their structures;

- Separate the fastener structure at the joint according to the unique " $\omega$ " linear shape of the fastener elastic strip, as shown in Figure6.

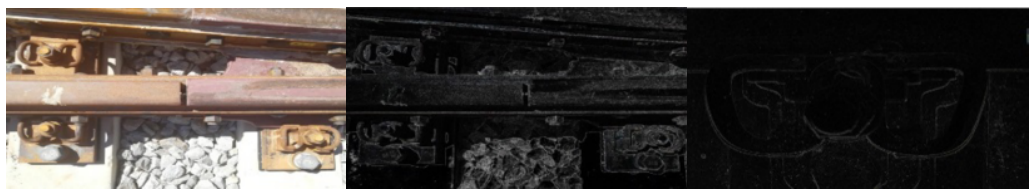

(a) Original image (b) Improve edge detection graph (c) Separate the fastener structure

Figure 6. Joint structure recognition and segmentation

It can be concluded from Figure 6 that the linear change of the track can be obtained in the turnout area. According to the linear alignment of the joint, the specific location of the joint can be obtained. Using the same principle, the track crack can be identified and processed. Compares Figure 6(b) with the sobel method, it can be concluded that the results of the elastic strips of the fasteners obtained by the improved edge detection recognition are better than that of the sobe, which can clearly separate and determine the location of the fasteners.

- Select rail joints, curved sections of rails, and sleeper photos for edge detection to identify their damage, as shown in Figure 7.

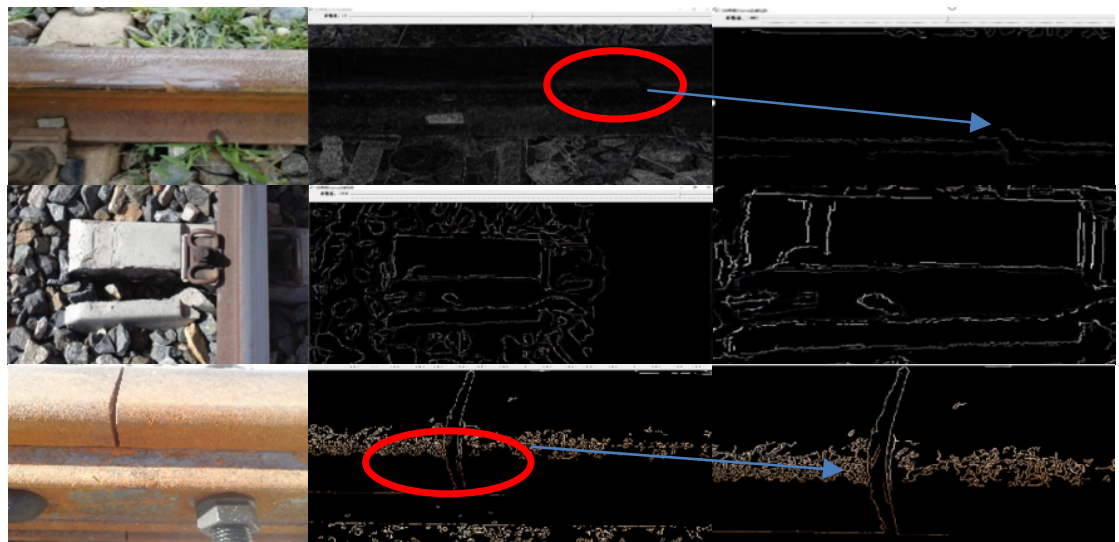

(a) Original image

(b) Improve edge detection graph (c) Details of edge detection in this paper

Figure 7. Damage edge detection

Comparing Figure 7(b), we can see that the results of improved edge detection have better effect on detailed structure screening than Canny edge detection, and have higher definition. From Figure 7(c), the line shape of improved edge detection is more obvious, and the rail outer edge is relatively straight, and the inner side has obvious linear sudden changes, which can clearly determine the phenomenon of rail surface falling; according to the large cracks and the increase of the sleeper edge line, the rail joint and sleeper damage can be identified. 
After the edge detection and processing, the edge image can already show the boundary line. Therefore, its geometric features are relatively intuitive. The geometric features can be extracted from the image to quantitatively analyze the diseases existing in the track structure. For the rail, the boundary line is in a straight line state, and no angle is formed in the damage-free state. Moreover, the steel rails perimeter is a fixed value, and the perimeter and area of the fasteners are fixed value in the original state. Analyze and process the images of rail and fastener collected by the line in operation and extract the geometric feature data in the image after edge detection by inactive railway. As shown in Table 2.

Table2. Image geometric feature data after edge detection

\begin{tabular}{cccccc}
\hline & Geometric feature & \multicolumn{2}{c}{ Inactive railway } & \multicolumn{2}{c}{ Operational railway } \\
\hline Steel & Angle $\left(^{\circ}\right)$ & 180.07 & 180.11 & 129.64 & 139.76 \\
rails & Perimeter $(\mathrm{cm})$ & 48.713 & 48.215 & 58.118 & 54.226 \\
\multirow{2}{*}{ Fasteners } & Area $\left(\mathrm{cm}^{2}\right)$ & 243.48 & 242.78 & 181.191 & 15.275 \\
& Perimeter $(\mathrm{cm})$ & 35.63 & 35.72 & 28.41 & 3.56 \\
\hline
\end{tabular}

The method in this paper is compared with the existing edge detection meehods by evaluating index PSNR(formula 4) and processing time. As shown in Table 3.

$$
I_{P S N R}=10 \cdot \lg \left(\frac{M A X_{I}^{2}}{I_{M S E}}\right)
$$

Where, MSE is the mean square deviation before and after image processing.

Table3. Comparison of image edge detection data

\begin{tabular}{cccc}
\hline Method & Sobel & Canny & This article \\
\hline PSNR & 51.31 & 59.87 & 62.37 \\
Time $(\mathrm{ms})$ & 842 & 804 & 768 \\
\hline
\end{tabular}

According to the comparison of time in Table 3, compared with the existing detection methods, it can be concluded that the PSNR of this algorithm is improved by $12.2 \%$, the processing time is shortened by $6.8 \%$ and the detection rate is improved.

\section{Conclusion}

- Through the equalization of the image histogram, the image gray scale is promoted. Thus the image contrast is reduced to a certain extent. For some areas with sharp gray scale changes, the frequency spectrum is more relaxed and the recognition of image filtering processing is improved;

- Using matrix determinant transformation processing, an improved two-dimensional convolution for image filtering is proposed. Compared with traditional detection methods, the detection speed can be increased by $12.47 \%$ and the resolution can be improved by $23.8 \%$;

- Combined improved edge detection with linear change, this method can identify specific track joints, separate the fastener structure and identify rail damage.Compared with the existing detection methods, the PSNR of this algorithm is improved by $12.2 \%$, the processing time is shortened by $6.8 \%$, 
which is feasible and practical in the daily operation and maintenance of the track.

\section{Acknowledgement}

The author thanks the tutor and his National Natural Science Foundation of China (51568037) for their help in this paper.

\section{References}

[1] Wei XL, Zhang ZH. Analysis and non-destructive testing of main diseases (defects) of ballastless track of high-speed railway. Railway Standard Design, 2011 (03):38-40.

[2] Li CH. Rail. Chengdu: Southwest Jiaotong University Press, 2012, 2-3.

[3] Zhu YT. Automatic identification method of track slab damage based on canny operator. Shanghai Railway Technology,2017(04):55-57.

[4] Wang XJ, Liu XM, et al. Image edge detection algorithm based on improved canny operator. Computer Engineering, 2012, 38(14):196- 198+202.

[5] Lopez-Molina C, Baets BD, Bustince H. A framework for edge detection based on relief function. Information Sciences, 2014,278(10):127-140.

[6] Wang BJ, Zhao HQ, Liu C. An improved edge detection algorithm based on canny operator. Innovation in Science and Technology, 2018(27): 13-14.

[7] Shih FY, Cheng SX. Automatic seeded region growing for color image segmentation. Image and Vision Computing, 2005,23(10): 877-886.

[8] Duan HY, Shao H, et al. An improved algorithm for image edge detection based on canny operator. Journal of Shanghai Jiaotong University,2016,50(12):1861-1865.

[9] Liu LX, Li BW, et al. Remote sensing image segmentation based on improved canny edge detection. Computer Engineering and Applications, 2019, 55(12): 54-58+ 180.

[10] Min YZ, Xiao BY, et al. Machine vision rapid detection method of the track fastener missing. Journal of Shanghai Jiaotong University, 2017, 51(10): 1268-1272.

[11] Yue B, Min YZ, MA HF, et al. Research on image enhancement preprocessing method in rail surface defects detection system. Journal of Railway Science and Engineering, 2018,15(12):3248- 3256.

[12] Yin H, Gong YH, Qiu GP. Fast and efficient implementation of image filtering using a side window convolutional neural network. Signal Processing, 2020,176.

[13] Wang N, Chen Y, Yao YL, Zhang Q, Jia LN, Gui ZG. Image smoothing via adaptive fourth-order partial differential equation model. The Journal of Engineering,2019,2019(11).

[14]Li YJ, Huang JB, Narendra A, Yang MH. Joint image filtering with deep convolutional networks. IEEE Transactions on Pattern Analysis and Machine Intelligence, 2019,41(8). 